\title{
TARATURA DI DUE GRAVIMETRI WORDEN E COLLEGAMENTI EUROPEI
}

\author{
Carlo Morelli
}

1. Premessa. - L'Istituto Nazionale di Geofisica possiede due gravimetri Worden (n. 50 e n. 52). Si tratta di strumenti il cui pregio principale è costituito, oltre che dalla grande manegevolezza e precisione delle misure, dalla cospicua estensione del campo di misura, che praticamente consente di effettuare collegamenti diretti fra le latitudini ed altitudini più disparate.

Ma perché questi collegamenti diretti fra punti con notevoli differenze di gravità abbia significato, è necessario che la costante di scala sia determinata con grande esattezza. Essa viene fornita dalla Casa costruttrice con un'approssimazione di circa il $2 \%$ : più che sufficiente per la prospezione geofisica, ma insufficiente per $i$ collegamenti a grande raggio sopra menzionati.

Anzi, già nei collegamenti a carattere regionale può rivelarsi la necessità di una maggiore sicurezza nella costante di scala. come è stato dimostrato per es. per il W. 50 ( ${ }^{1}$ ).

$\mathrm{Ne}$ consegue quindi l'opportunità di procedere alla determinazione della predetta costante con la massima sicurezza possibile.

Non potendo essere questa fornita né dal metodo del piano inclinato, né dalle misure sulla verticale $\left({ }^{2}\right)$, è indispensabile ricorrere alle misure pendolari : queste acquistano quindi nuova importanza (e praticamente vengono continuate quasi solo per questo motivo, dato che - come vedremo - per tutte le altre applicazioni esse possono essere vantaggiosamente sostituite dai gravimetri, con opportuni accorgimenti e cautele).

Senonché le misure pendolari, almeno quelle esistenti fino a pochi anni fa, non possono garantire in generale la precisione richiesta: raramente si può essere sicuri del mgal, e ben spesso sono possibili errori anche di parecchi mgal! La ragione di ciò va attribuita ad un gran numero di cause, di cui alcune ben note (e fra queste spesso la principale è l'imperfetta determinazione del tempo), mentre altre molto spesso sfuggono al controllo dell'osservatore (microsismi, effetti magnetici, ecc.). 
Praticamente solo dopo l'abbandono dell'antico relais delle coincidenze e l'introduzione della registrazione fotografica per la determinazione dei periodi ( $1937 \mathrm{ca}$ ), è stato possibile raggiungere nelle misure pendolari una precisione dell'ordine di $0,5 \mathrm{mgal}$. Questa viene oggi assicurata in America con i pendoli della Gulf, in Inghilterra con quelli del Dept. of Geodesy and Geophysics di Cambridge, ed è stata raggiunta in Germania prima della seconda guerra mondiale ad opera dell'Istituto Geodetico di Potsdam.

Come risulta infatti da una pubblicazione recente del WeIKEN $\left({ }^{3}\right)$, prima e durante la guerra sono state eseguite in Germania e nei territori allora occupati ben 171 stazioni pendolari di riferimento divise in 16 distretti, tutte collegate più volte con Potsdam, in generale da osservatori diversi, con strumenti diversi, in anni differenti. Alcune di queste sono collegate addirittura una decina di volte con Potsdam (per es. Weidenau e Karlsruhe: v. tab. II; ed in particolare la base tedesca di taratura dei gravimetri Harzburg-Torfhaus). Il più sicuro collegamento con Potsdam è quello di Harzburg (22 collegamenti! errore medio $\div 0,13 \mathrm{mgal}$ ).

La fig. 1 riporta il quadro di queste stazioni nell'attuale Germania occidentale, rappresentate a seconda dei rispettivi errori medi.

In essa è anche indicata la catena di queste stazioni che è stata scelta per la taratura. Come si vede dalla fig. 2 , essa abbraccia un campo di circa $1500 \mathrm{mgal}$, nel quale sono comprese:

a) quasi tutta la regione corrispondente alle latitudini (gravimetriche) dell'Europa centro-meridionale; in particolare l'Italia;

b) molte delle stazioni nazionali di riferimento più importanti;

c) tutte le misure assolute moderne (ad eccezione di Leningrad, in cui valore definitivo però non è noto).

Nella catena di taratura è stato incluso il maggior numero di stazioni sicure, onde aver la possibilità di una compensazione che consentisse di giudicare dell'attendibilità reale delle misure pendolari (v. $\$ 4$ j.

Le misure sono state eseguite nei mesi di agosto e settembre 1951 , e precisamente: dall'll al 20 agosto da Villaco ad Uccle; dal 21 agosto al 3 settembre (durante la IX Assemblea Generale dell'Unione Geodetica e Geofisica Internazionale) i collegamenti internazionali da Uccle; dal 4 al 9 settembre da Euskirchen a Flensburg; dal 10 al 30 settembre il ritorno Flensburg-Euskirchen-Villaco; il 4.6 agosto e l'l-3 ottobre i collegamenti Villaco-Trieste e Trieste-Padova. 


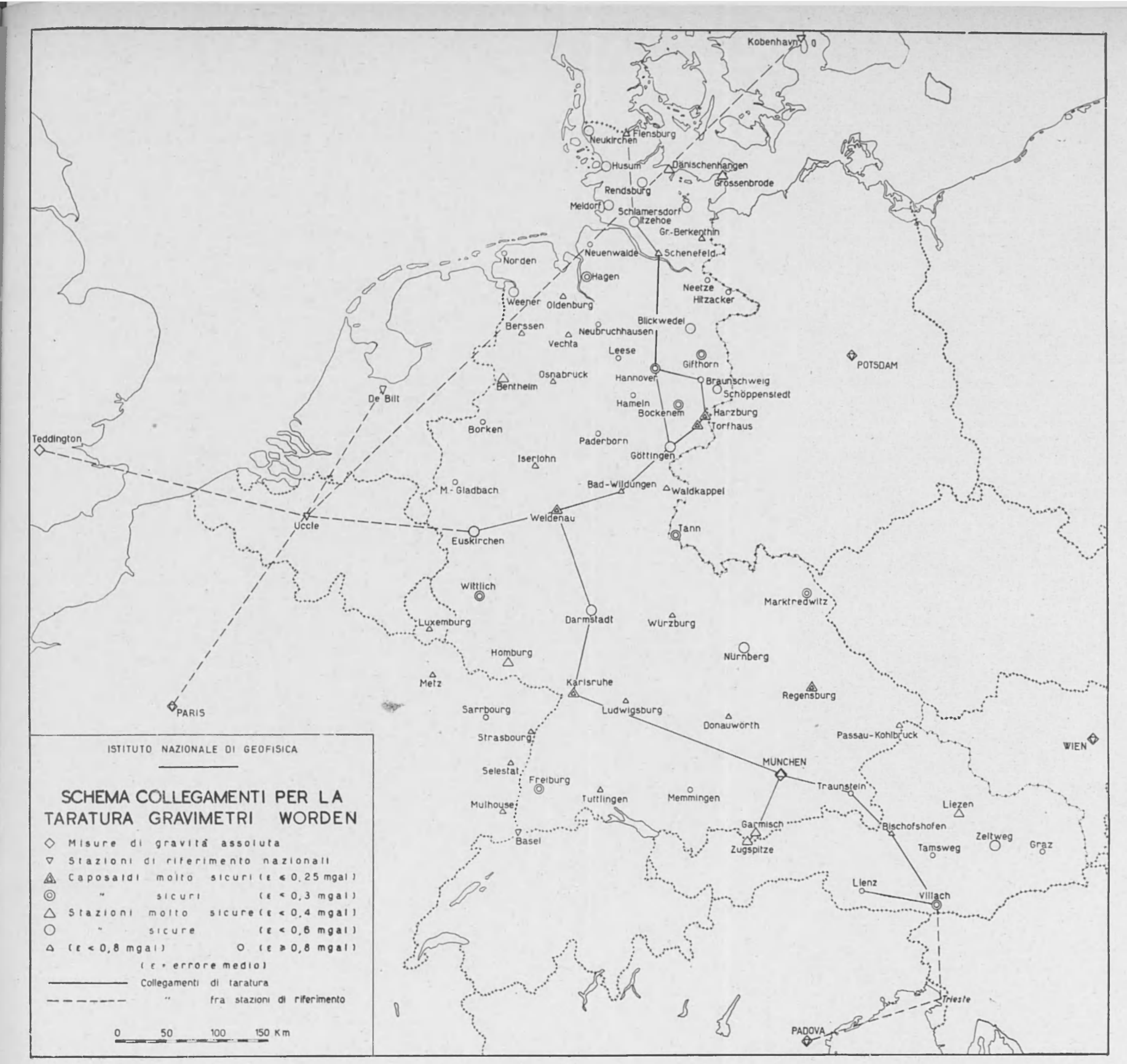




\section{Parte I. - TARATURA DeI gRaVimetri}

2. Esecuzione delle stazioni, criteri d'impiego e di riduzione. a) Poiché, com'è noto $(-)$, i gravimetri Worden hanno deriva, e non sempre questa è costante, il criterio fondamentale d'impiego è stato quello di eseguire tutte le misure in modo da poter ritornare al più presto al punto di partenza, cosi da poter controllare sia la deriva che eventuali salti (che però non sono stati mai riscontrati). Perciò le singole tratte sono state scelte in modo che gli estremi non distassero più di qualche ora di macchina $\left({ }^{*}\right)$, e ogni tratta $A B$ è stata eseguita col "passo del pellegrino" $(A \cdot B \cdot A \cdot B)$, sia in andata che al ritorno. Ogni tratta è stata quindi percorsa in generale 6 volte; e se le tre tratte (in andata e ritorno) si potevano eseguire tutte nella stessa giornata, allora ne risultavano due collegamenti con lo stesso peso $(A \cdot B \cdot A ; B \cdot A-B)$; altrimenti, veniva determinato e tolto il contributo della deriva notturna (in -genere, ben diversa da quella diurna durante il trasporto e l'impiego dello strumento) ed ai collegamenti così risultanti si attribuiva peso metà.

Un numero di volte ancor maggiore sono stati eseguiti i collegamenti fra Harzburg, Euskirchen (ed Uccle), dato che da questi dipendevano poi i collegamenti internazionali con Potsdam; e quelle fra Villaco, Trieste e Padova, stazione nazionale di riferimento italiana ( $v$ fig. I e Parte II). Invece constano di due collegamenti indipendenti la diramazione Monaco-Zugspitze e la tratta Hannover-Schenefeld, e di uno solo l'estremo settentrionale Schenefeld-Flensburg e la diramazione Villaco-Lienz.

Tutti i collegamenti sono stati eseguiti con autovettura appositamente attrezzata, e si è cercato di eseguire sempre le due tratte di ogni collegamento il più possibile nelle stesse condizioni (di durata, di temperatura, di sollecitazioni meccaniche, ecc.).

b) Nella grande maggioranza dei casi le stazioni pendolari sono state individuate con esattezza $\left({ }^{3}\right)$, grazie anche alle descrizioni supplementari gentilmente inviate dall'Istituto Geodetico di Potsdam e spesso per il ricordo di persone sul posto; solo in qualche caso (Lienz, Karlsruhe, Bad Wildungen, Flensburg) il posto esatto non era precisabile nell'ambito del locale, ma allora si sono sempre verificate le differenze fra tutte le posizioni possibili, ed esse non si sono mai trovate superiori a $0,05 \mathrm{mgal}$ (per l'influsso della diversità delle masse vicine). Inoltre, nelle stazioni pendolari o nelle immediate vicinanze

(*) Favoriti in ciò dall'ottima rete di autostrade tedesche. 


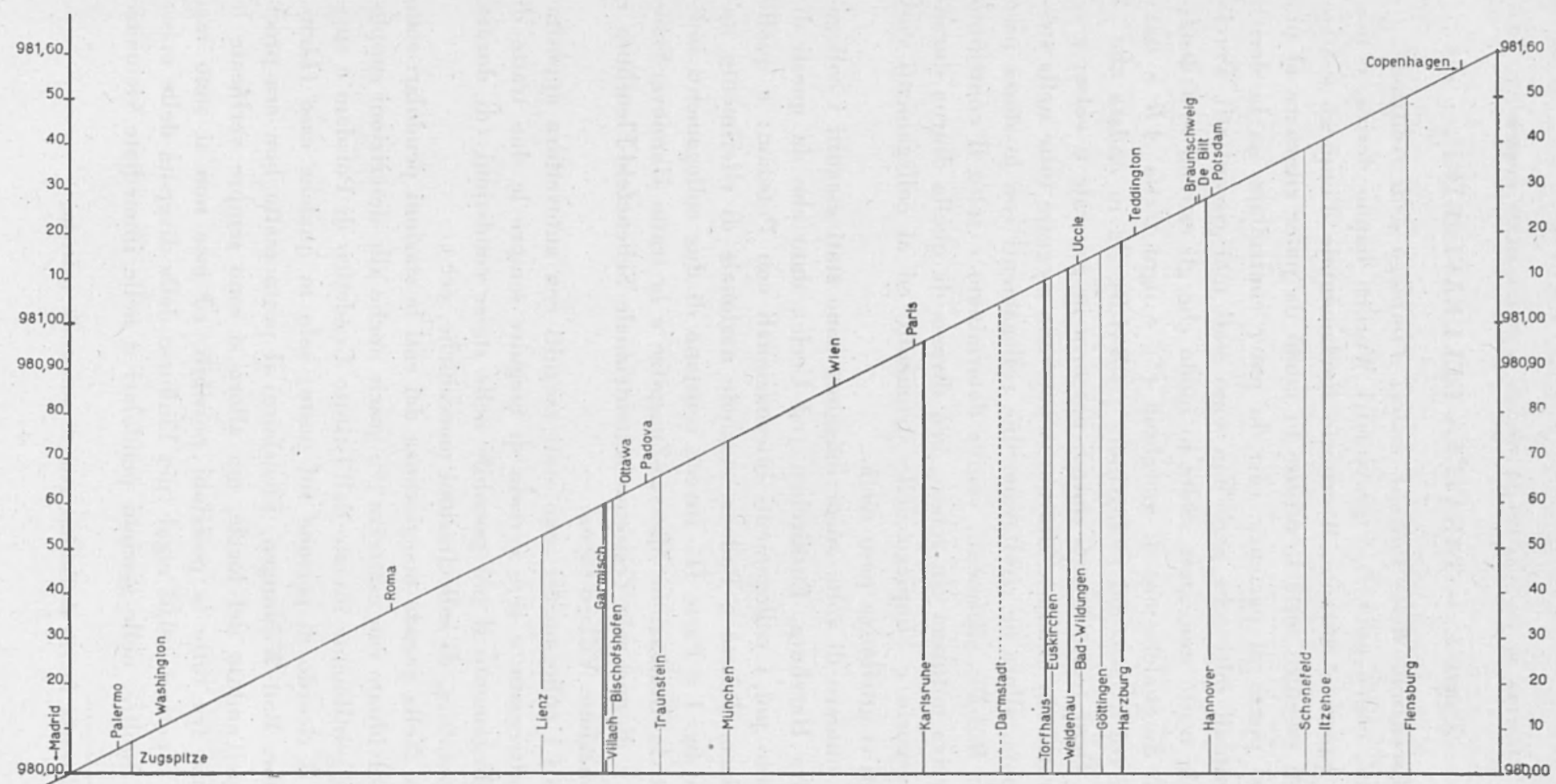

Fig. 2 - Estensione del campo di taratura, e stazioni assolute e di riferimento in esso comprese. 
non sono state mai riscontrate modifiche nelle opere murarie o sul terreno tali da alterare in maniera sensibile il valore della gravità.

Dai calcoli per la taratura è stata poi esclusa la stazione di Darmstadt, in quanto l'antica cappella nel parco del castelletto Principe Emilio è stata distrutta, come tutto il castelletto; nella ricostruzione, il pavimento è stato rifatto in cemento e rialzato.

c) Poiché, data la precisione delle misure, la variazione diurna della gravità non è più trascurabile, di essa si è tenuto previamente conto nella riduzione di tutte le misure. Essa è stata dedotta dai diagrammi della variazione diurna della gravità nell'Italia sett. pubblicati mensilmente dall'Osservatorio Geofisico di Trieste, calcolati teoricamente nell'ipotesi di una Terra rigida ed amplificati del fattore $1,27+0,10$ ricavato sperimentalmente per il rapporto fra le ampiezze della marea osservata e di quella teorica $\left({ }^{4}\right)$. Ai dati validi per l'Italia sett. sono state apportate le debite correzioni (molto piccole) per tener conto delle differenze di latitudine (influenza sull'ampiezza) e di longitudine (influenza sulla fase).

Per le giornate di misura a Parigi (1-2 sett.) tale variazione diurna è stata confrontata con quella (teorica) calcolata per cortesia del prof. Martin dell'Office de la Recherche Scientifique Outre-mer, ed è stata trovata in ottimo accordo.

3. Risultato delle osservazioni. Precisione. - I risultati delle osservazioni sono riportati nella tab. I.

Si vede da questa anzitutto che l'errore medio delle misure con gravimetro - prescindendo dall'eventuale errore nella costante di scala - è in generale inferiore a $0,1 \mathrm{mgal}$, e solo in rari casi può raggiungere i $0,2 \mathrm{mgal}$; e vedremo più avanti (\$ 4) che il significato di questi errori medi è reale, e non apparente come molto spesso nel caso di quelli per le misure pendolari.

La realtà di questa precisione risulta anche per esempio dal confronto fra i risultati del collegamento Göttingen-IIannover, eseguito sia direttamente che attraverso la base di taratura Harzburg-Torfhaus e la stazione di Braunschweig:

\begin{tabular}{|c|c|c|}
\hline Gottingen-Hannover & $W .50$ & W. 52 \\
\hline $\begin{array}{l}\text { via: diretta }: \dot{\mathbf{B}}:-\dot{0} \\
\text { via: } \mathbf{T} \cdot \mathbf{H}=\end{array}$ & $\begin{array}{r}+120.55 \\
.54\end{array}$ & $\begin{array}{r}+120.82 \\
.79\end{array}$ \\
\hline..... & $+\quad 0.01$ & $+\quad 0.03$ \\
\hline
\end{tabular}


Tabella I - Risultati delle osservazioni (in mgal).

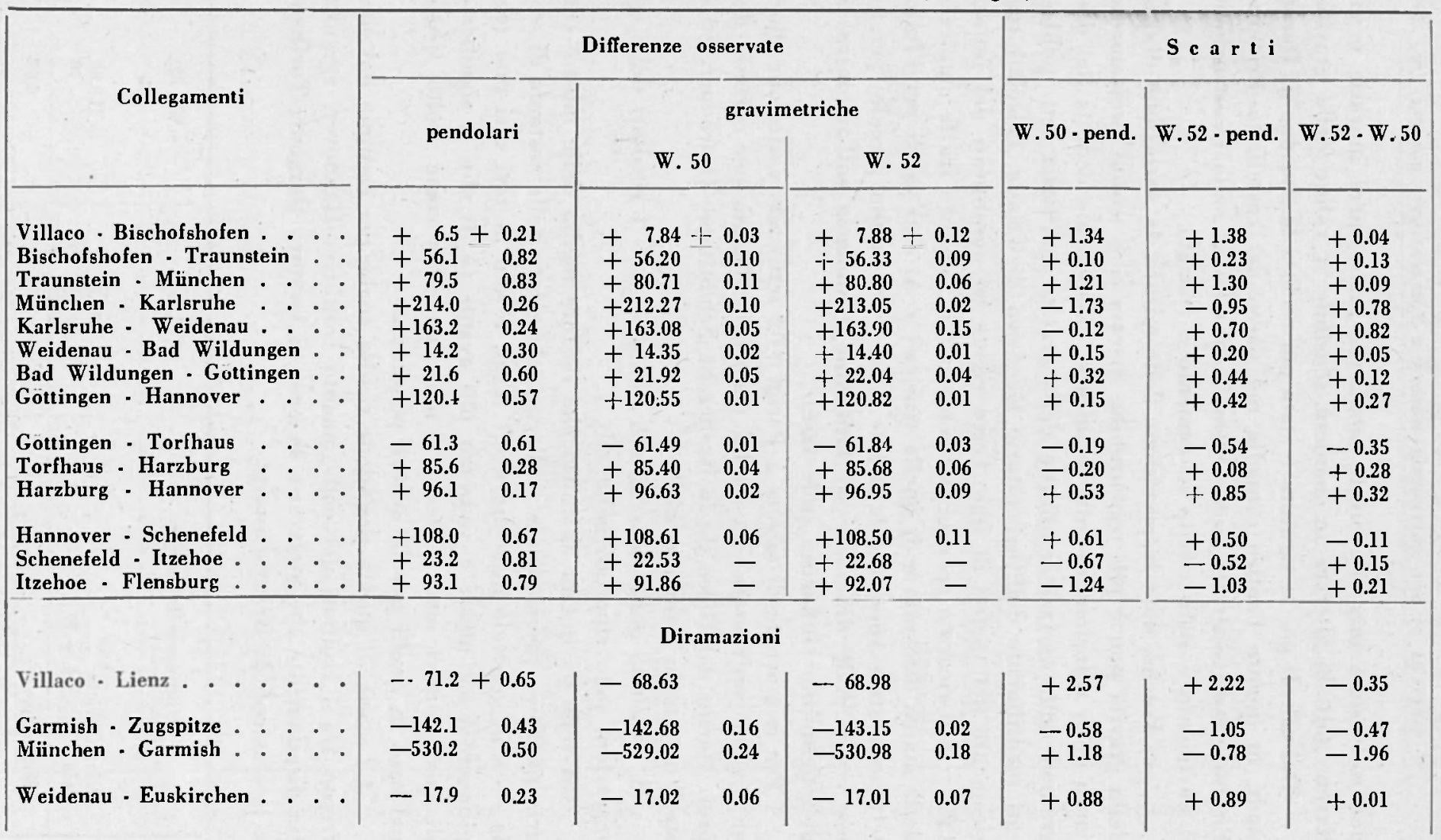


Per mettere però meglio in evidenza la natura sistematica degli scarti fra misure gravimetriche e misure pendolari, conseguenti ad errori nelle costanti di scala dei due gravimetri, riportiamo nella tab. II i valori delle stazioni pendolari, ordinate per valori crescenti della gravità, ed in corrispondenza le differenze da questi dei valori ricavati con i due gravimetri, assumendo come punto di partenza $(\Delta=0)$ la stazione di Villaco.

Gli stessi dati sono riportati nella fig. 3 , dove si vede chiaramente l'influsso degli errori di natura sistematica ora menzionati.

Dalla stessa figura si vede anche che, a prescindere da questi errori sistematici, i due diagrammi $W .50$ e W. 52 presentano una similitudine perfetta: altra prova della buona precisione delle misure gravimetriche (su cui ritorneremo al $\S 5$ ). Gli scarti dall'andamento lineare sono da ritenersi quindi per la massima parte dovuti agli errori delle misure pendolari.

Gli errori di natura sistematica dei due gravimetri sono di segno contrario: cioè, la costante di scala del gravimetro 52 era affetta da un errore sistematico per eccesso, quella del n. 50 di un errore sistematico per difetto. L'errore percentuale risulta in media dal rapporto

$$
\frac{\Delta_{52}}{\Delta_{50}}=+\Delta_{50}, 0040+0.0007 .
$$

L'errore (relativo) fra i due strumenti è quindi del 4\%o: dell'ordine cioè di quello menzionato nel $\S 1$, e troppo forte per poter essere trascurato (nell'impiego geodetico).

La media degli errori medi pendolari per le differenze di gravità che interessano la presente taratura è invece $+(0,49+0.06)$ mgal.

4. Calcolo delle nuove costanti strumentali. - L'esame della fig. 3 mostra chiaramente che, a meno degli errori connessi con le misure pendolari, l'andamento degli scarti fra le misure con ciascuno dei due gravimetri e quelle pendolari è lineare, per cui potrà essere determinato con sufficiente approssimazione mediante una funzione del tipo

$$
a x+b y+k=0 .
$$

Quali valori di $b$ e di $k$ si sono assunti quelli della tab. II, previa sottrazione dai primi del valore 980,000 . Escludendo la stazione di Villaco, dove (fig. 3) lo scarto della stazione pendolare è manifestamente troppo forte, si sono ottenuti due sistemi di 17 equazioni gene- 
Tabella II - Differenze (progressive) fra misure gravimetriche e pendolari (punto di partenza: Villaco).

\begin{tabular}{|c|c|c|c|c|c|}
\hline \multirow{3}{*}{ N. Stazione } & \multicolumn{2}{|l|}{$b$} & \multirow{3}{*}{$\begin{array}{l}\text { Numero } \\
\text { colleg. pend. }\end{array}$} & \multicolumn{2}{|c|}{$--k$} \\
\hline & \multirow{2}{*}{\multicolumn{2}{|c|}{$g$ pendolare }} & & \multicolumn{2}{|c|}{ Differenze（mgal) } \\
\hline & & & & W. 52 & W. 50 \\
\hline 1 Zugspitze & $980,070.8 \div$ & 0.35 & 3 & +3.25 & +1.08 \\
\hline 2 Lienz & ,529.8 & .65 & 2 & +2.57 & +2.22 \\
\hline 3 Garmisch & ,601.0 & .36 & 6 & +2.07 & +1.86 \\
\hline Villaco & ,601.0 & .05 & 3 & 0.00 & 0.00 \\
\hline 4 Bischofshofen &, 607.5 & .20 & 2 & +1.34 & +1.38 \\
\hline 5 Traunstein & ,663.6 & .79 & 2 & +1.44 & +1.61 \\
\hline 6 München &, 743.1 & .24 & 4 & +2.65 & +2.91 \\
\hline 7 Karlsruhe & ,957.1 & .09 & 16 & +0.92 & +1.96 \\
\hline 8 Torfhaus & $981,094.8$ & .25 & 10 & +1.08 & +2.76 \\
\hline 9 Euskirchen & , 102.4 & .07 & 2 & +1.68 & +3.55 \\
\hline 10 Weidenau & , 120.3 & .22 & 10 & +0.80 & +2.66 \\
\hline $11 \mathrm{~B}$. Wildungen & ,134.5 & .20 & 2 & +0.95 & +2.86 \\
\hline 12 Gottingen &, 156.1 & .56 & 4 & +1.27 & +3.30 \\
\hline 13 B. Harzburg &, 180.4 & .13 & 22 & +0.88 & +2.84 \\
\hline 14 Hannover &, 276.5 & .11 & 4 & +1.42 & +3.72 \\
\hline 15 Schenefeld & ,384.5 & .66 & 3 & +2.03 & +4.22 \\
\hline 16 Itzehoe &, 407.7 & .47 & 3 & +1.36 & +3.70 \\
\hline 17 Flensburg &, 500.8 & .64 & 3 & +0.12 & +2.67 \\
\hline
\end{tabular}




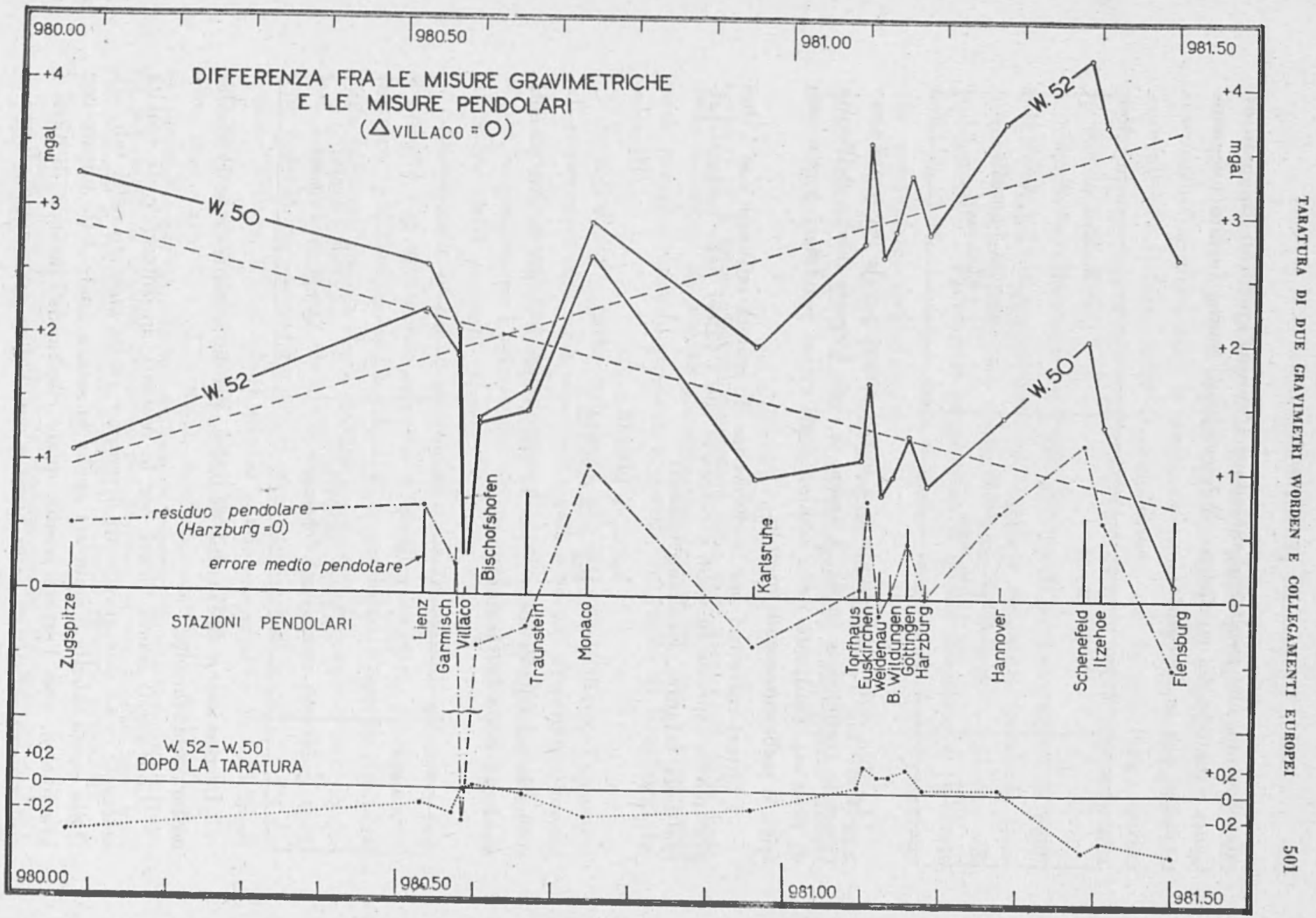

Fig. 3 
rate ciascuno, che risolti col metodo dei minimi quadrati (metodo di Gauss e metodo dei coefficienti indeterminati) hanno fornito i seguenti valori per le incognite:

\begin{tabular}{|c|c|c|}
\hline & W. 50 & W. 52 \\
\hline $\begin{array}{c}\mathbf{x} \\
\mathbf{y} \\
\mathbf{m}_{0}\end{array}$ & $\begin{array}{c}+2,959+0,373 \\
-0,001481+0,000360 \\
+-0,546 \mathrm{mgal}\end{array}$ & $\begin{array}{l}+0,818 \quad+0,347 \\
+0,001900+0,000337 \\
+00,512 \mathrm{mgal}\end{array}$ \\
\hline
\end{tabular}

Le due rette corrispondenti a questi valori per le incognite sono riportate tratteggiate in fig. 3. Come si vede, l'errore medio dell'unità di peso $m_{0}$, risultante della somma degli errori pendolari e gravimetrici, è sufficientemente piccolo.

I fattori correttivi per le differenze di gravità misurate con i due gravimetri, espresse in mgal e calcolate con $i$ valori delle costanti prima della taratura, risultano quindi :

$$
\begin{aligned}
& k_{50}=+1,00148 \\
& k_{52}=+0,99810
\end{aligned}
$$

sicché le costanti di scala $c_{1}$ per la vite grande e $c_{\text {o }}$ per la vite piccola risultano dopo la taratura:

\begin{tabular}{|c|c|c|}
\hline \multirow{2}{*}{ costante } & W. 50 & W. 52 \\
\hline & & \\
\cline { 2 - 3 } c $_{1}$ & $+4,31739+0,00155$ & $+5,84567+0,00197$. \\
$c_{2}$ & $+0,99082+0,00036$ & $+1,00359-0,00034$ \\
\hline
\end{tabular}

La precisione dell'ordine di $0,35 \%$ per le costanti di scala risulta molto soddisfacente.

Con questi nuovi valori per le costanti, le differenze di gravità osservate con i due gravimetri diventano quelle indicate nella tab. III. Nella stessa tabella è riportata anche la media finale delle misure con gravimetro, con l'errore medio quale risulta dall'insieme di tutti i collegamenti effettuati (con l'uno e con l'altro gravimetro). Come si 
verle, questo errore medio è in generale molto piccolo (valore medio: $0,10+0,01 \mathrm{mgal}$ ) e non supera mai $0,2 \mathrm{mgal}$ (ad eccezione del tratto Garmisch-Zugspitze, dove le condizioni sono state particolarmente sfavorevoli per il forte salto di temperatura, per la più lunga durata della misura dovuta agli orari della funicolare, e per il trasporto stesso con la funicolare).

Anche le differenze fra $i$ due gravimetri si mantengono in generale entro i limiti sopraddetti, ad eccezione del tratto terminale Hannover-Schenefeld, che però consta - come si è detto — di due soli collegamenti. L'andamento progressivo di queste differenze è riportato nella fig. 3 (in basso), e come si vede è in generale (con l'eccezione ora menzionata) soddisfacente.

Prendendo allora per base il valore pendolare di $g$ a Harzburg, quale risulta ( $\left.{ }^{3}\right)$ dalla media di 22 collegamenti indipendenti diretti con Potsdam:

$$
\mathrm{g}_{\text {IIarzluarg }}=981,180.4+0.13
$$

i valori di $g$ nelle stazioni pendolari sono stati ricalcolati sulla base delle differenze di gravità ottenute con gravimetro, con i loro errori medi progressivamente crescenti a partire da quello di Harzlourg (tabella III).

Come si vede, anche nei punti più distanti (Villaco, Zugspitze) tali errori medi non superano i $0,4 \mathrm{mgal}$; e quindi dimostrano ancora una volta la possibilità dell'impiego di questi gravimetri a scopo geodetico, e giustificano i criteri che hanno guidato l'impostazione e l'esecuzione della presente ricerca.

Ammettendo in prima approssimazione trascurabili gli errori delle misure con gravimetro rispetto a quelli pendolari, potremo considerare la differenza fra $i$ valori di $g$ pendolari e quelli risultanti dalla presente ricerca come dovuta essenzialmente agli errori pendolari. Essa ci dice allora (tab. III) che tutte le stazioni pendolari tedesche considerate presentano errori non supericri al mgal (*). Fa eccezione Villaco, il cui errore medio pendolare sarebbe $\div 0,05 \mathrm{mgal}$ (tre collegamenti indipendenti, anni 1939 e 1940) e che invece presenta uno scarto di $+1,76 \mathrm{mgal}$. Anche ammettendo che una parte di questo sia dovuta alla misura con gravimetri, la discrepanza è troppo più forte di quanto ammesso dal piccolo errore medio, per non far pensare a qualche errore sistematico nelle misure pendolari.

(*) Conglobando in questi anche le eventuali piccole differenze per diversità nel posto di misura (v. $\$ 2, b$ ). 
Tabella III - Differenze di gravità dopo la taratura e valori di g.






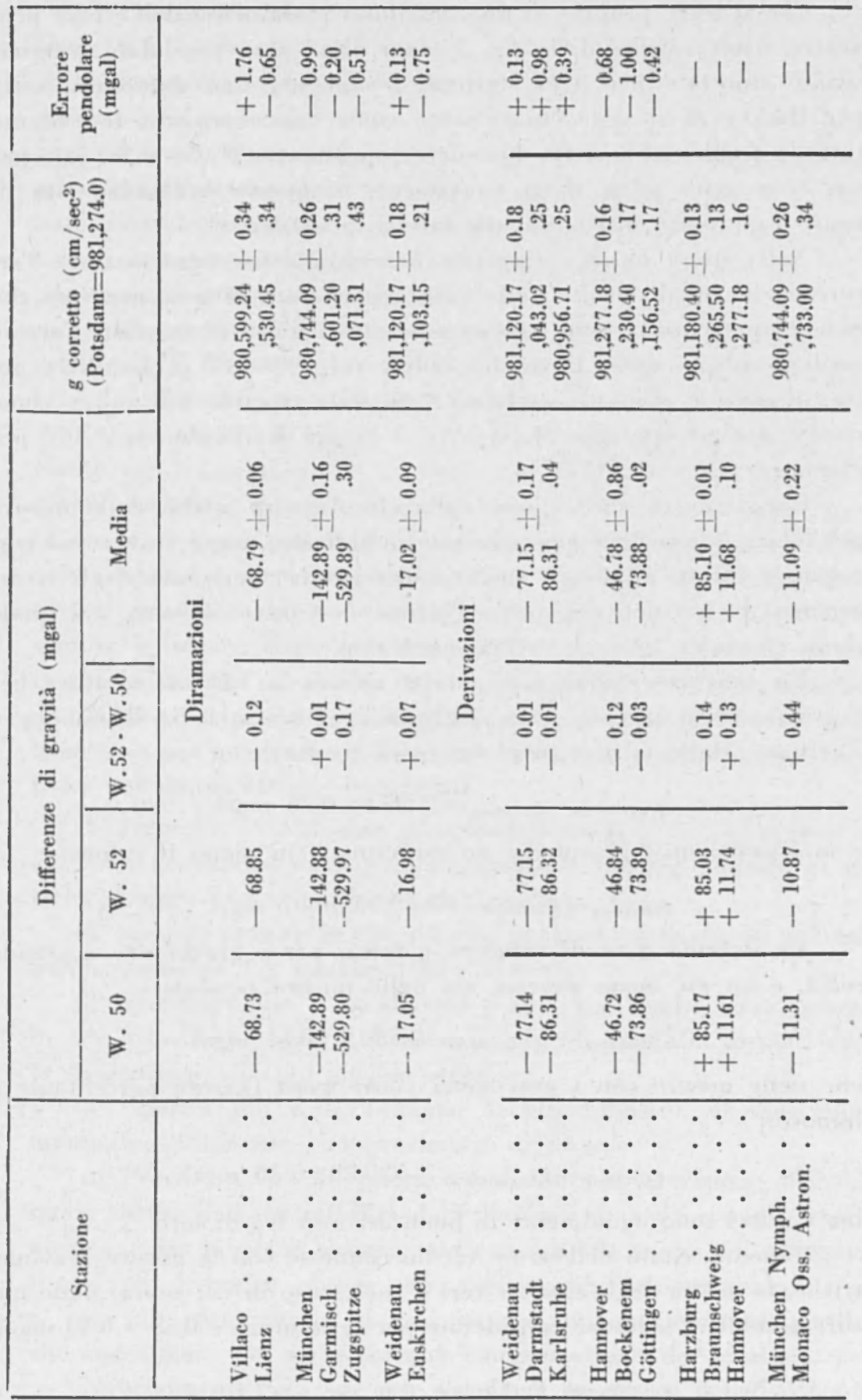


Che si tratti proprio di un contributo preminente dell'errore pendolare risulta anche dalla fig. 3 , dove il residuo pendolare progressivo è riportato per ogni stazione, assumendo una differenza nulla per Harzlurg: si vede chiaramente come esso riproduca fedelmente tutte le accidentalità delle due curve soprastanti (W. 50 e W. 52 ); per cui la maggior parte degli scostamenti di queste dall'andamento lineare va proprio attribuita alle misure pendolari.

Nella stessa fig. 3 è riportato in ordinate per ogni stazione l'errore medio, quale risulta dalle misure pendolari. Si può osservare che non sempre l'errore vero è compreso entro i limiti di tre volte l'errore medio (entro i quali dovrebbe cadere col $999 \%$ di probabilita), anche quando le stazioni pendolari sono state ripetute più volte: come risulta per esempio per Monaco $(n=4)$, per Karlsruhe $(n=16)$, per Hannover $(n=4)\left({ }^{* *}\right)$.

Ciò dimostra ancora una volta che l'errore medio delle misure pendolari, quale viene generalmente pubblicato, non è reale; esso corrisponde infatti all'errore medio " intrinseco", derivante dagli errori accidentali, ma non rispecchia l'errore "estrinseco ", vero, dal quale siano eliminati tutti gli errori sistematici.

Un confronto particolare merita ancora la base di taratura per i gravimetri a piccola scala, istituita in Germania fra Harzluurg e Torfhaus. Dalle misure pendolari essa risulta:

$$
\mathrm{g}_{\text {Harzburg }}-\mathrm{g}_{\text {Torrhani: }}=+85.6 \div 0.28 \mathrm{mgal} \text {, }
$$

e le misure con i gravimetri ne confermano in pieno il valore:

$$
\mathbf{g}_{\text {Harzburg }}-\mathbf{g}_{\text {Torfhaus }}=+85.53 \div 0,07 \mathrm{mgal} \text {. }
$$

La seconda base di taratura tedesca, per i gravimetri a grande scala, è un po' meno precisa, sia nelle misure pendolari:

$$
\mathrm{g}_{\text {Garmisch }}-\mathrm{g}_{Z \text { ugspitze }}=+530.2 \div 0,51 \mathrm{mgal}
$$

che nelle misure con i gravimetri (dove però l'errore percentuale è minore):

$$
\mathrm{g}_{\text {Garmisch }}-\mathrm{g}_{\text {Zugspitze }}=+529.89+0,30 \mathrm{mgal}
$$

ma $\mathrm{i}$ valori sono ugualmente in buon accordo fra di loro.

Tenendo conto dell'errore medio connesso con le misure gravimetriche, la media degli errori " veri " (nel senso di cui sopra) delle misure pendolari tedesche considerate per la taratura è $0,53 \div 0,10 \mathrm{mgal}$.

(**) Non si include qui Euskirclicn, dato che $n=2$. 
5. Precisione delle misure con gravimetro Worden. Discussione. Come si è visto nel § precedente, purché convenientemente tarati e giudiziosamente impiegati, $i$ gravimetri Worden (e ciò vale, con le modifiche di circostanza, anche per altri gravimetri geodetici) possono oggi in determinate circostanze sostituire $i$ pendoli, e con vantaggio: tale anzi da offrire, qualora siano avverate le premesse ora menzionate, una sicurezza che solo difficilmente, e con dispendio di tempo e costi hen maggiori, i pendoli possono garantire. Discutiamo ora separatamente queste affermazioni.

a) Taratura. - Una taratura sicura può essere effettuata solo su una serie di stazioni pendolari del massimo affidamento. Ciò perché hen difficilmente si può oggi raggiungere con le misure pendolari (v. § 1) la precisione di $0,5 \mathrm{mgal}$, e anche questa non è sempre sufficiente per la taratura dei gravimetri a grande scala da impiegare a scopo geodetico. Allora solamente con più stazioni si potrà sperare in una compensazione degli errori pendolari, e solo con una serie di valori pendolari sicuri opportunamente distribuiti nel campo di taratura si potrà controllare l'andamento della costante di scala (che non sempre è lineare come nei due gravimetri qui esaminati, ma potrà essere facilmente parabolico, con curvature più o meno accentuate).

Ragioni di prudenza consiglieranno poi, ed in particolare nell'ultimo caso ora menzionato, di non estrapolare l'impiego dei gravimetri fuori del campo in cui sono tarati.

b) Impiego. - Abbiamo già specificato sopra $(\$ 2, a)$ a quali condizioni i gravimetri del tipo qui esaminato possano portare ai risultati richiesti. Esse si possono cosi riassumere:

$1^{\circ}$ operare sempre in circuiti che possano essere chiusi nel minor tempo possibile (al massimo, $10 \div 12$ ore);

$2^{\circ}$ procurare che le condizioni d'impiego (temperatura, vibrazioni per il trasporto, ecc.) si mantengano il più possibile costanti durante l'esecuzione di ogni singolo circuito;

$3^{0}$ ripetere più volte (almeno 3 ; possibilmente 4) ogni collegamento, possibilmente con gravimetri diversi.

c) Precisione. - In queste condizioni, la precisione del $0,5 \%$, quale risulta dall'attendibilità delle costanti di scala e dagli errori di misura, potrà ritenersi sicuramente conseguibile anche nei collegamenti a grandi distanze; ed essa potrà essere anche minore qualora si operi con più gravimetri e si eseguiscano poligonazioni gravimetriche, che consentano poi un'opportuna compensazione dei risultati.

Naturalmente ciò vale per l'impiego geodetico dello strumento; 
a scopo geofisico, quando cioè si opera senza la vite grande ed a distanze ravvicinate (che consentano la chiusura dei circuiti entro tempi molto brevi) la precisione del gravimetro Worden è dell'ordine di 0,03 mgal $\left({ }^{2}\right)$.

d) Conclusioni. - A conclusione di questa prima parte, possiamo dire che $i$ due gravimetri Worden n. 50 e n. 52 sono soddisfacentemente tarati nell'intervallo di circa $1500 \mathrm{mgal}$, da 980,00 a 981,50. In quest'intervallo, che abbraccia quasi tutta l'Europa centro-meridionale, un giudizioso impiego degli strumenti li raccomanda con vantaggio in sostituzione dei pendoli, e può garantire una precisione molto elevata.

6. Appendice sulle stazioni di taratura tedesche. - Indirettamente, dal lavoro qui sopra esposto è risultata controllata la serie di stazioni pendolari tedesche scelte per la taratura, facilitando così il compito per eventuali altre operazioni analoghe. Per questo motivo, riportiamo nella tab. IV qualche particolare più dettagliato per ciascuna di esse, che completi la descrizione pubblicata in $\left({ }^{3}\right)$ e consenta di rintracciare più facilmente il posto in cui sono state effettuate le misure con gravimetro.

Oltre alle stazioni pendolari utilizzate per la taratura, sono state effettuate misure con $i$ gravimetri anche a:

- Darmstadt, esclusa dalla taratura per i motivi sopra detti $(\$ 2, b)$;

- Bockenem, inclusa in un primo tempo e poi esclusa perché accessibile per strade dal fondo disagiato;

- Monaco, Osservatorio Astronomico, pilastro della misura assoluta; nonché a:

- Braunschweig, dove attualmente è in preparazione una nuova misura assoluta di gravità, a cura del dr. Rieckmann, col metodo della caduta dei gravi.

I risultati sono riportati in appendice alla tab. III.

La misura assoluta a Monaco (von Orff, 1887) aveva dato il risultato 980,736. La differenza col valore nell'attuale sistema di Potsdam sarebbe quindi di $+3,0$ mgal: questa misura assoluta sembra quindi molto imprecisa (v. $\$ 10$ ). 
TABELla IV - Dati descrittivi complementari sulle stazioni di taratura, e su altre stazioni, effettuate in Germania.

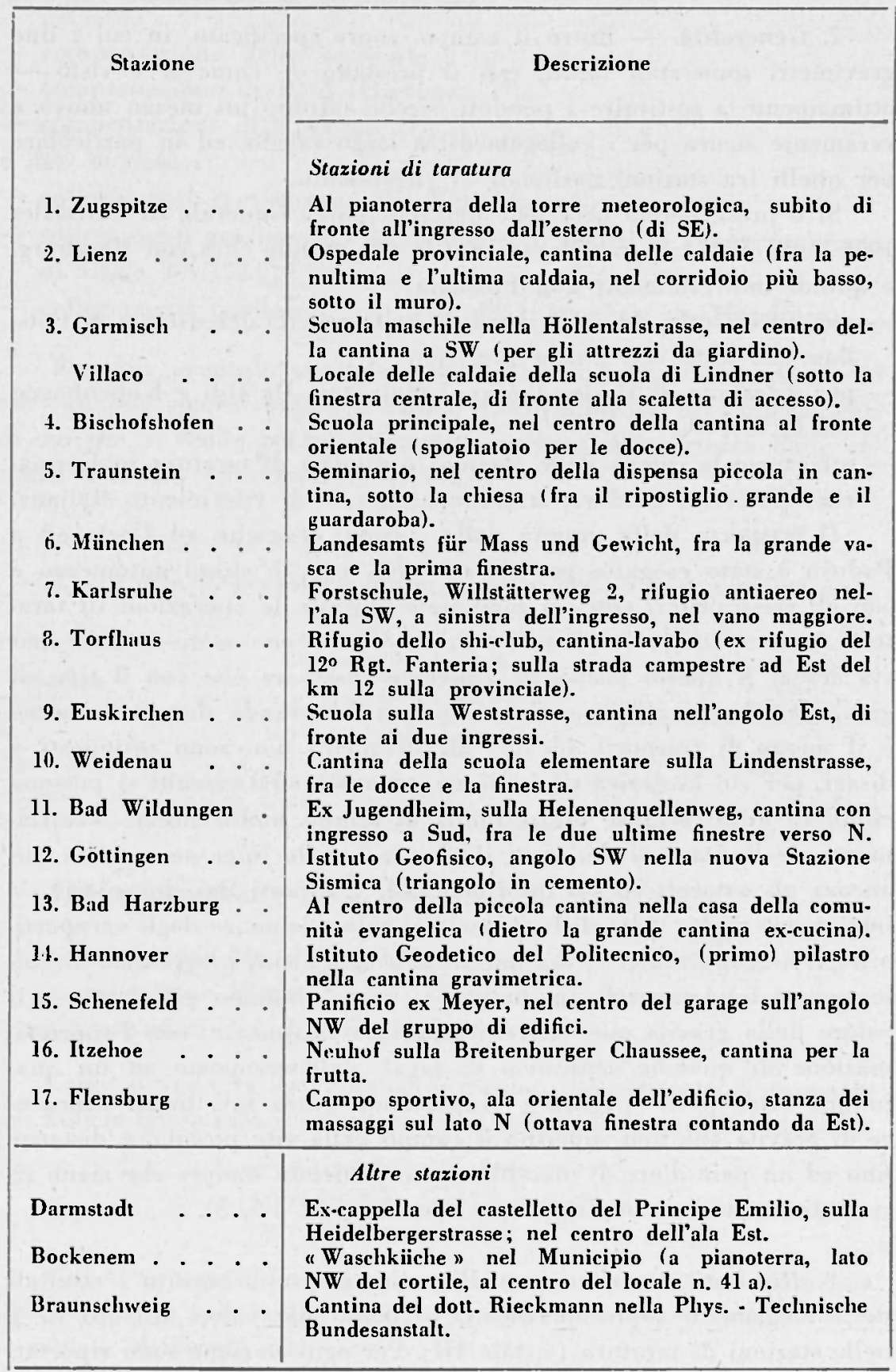




\section{Parti. II. - COLLEGaMenti EUROPEI}

7. Generalità. - Entro il campo, sopra specificato, in cui i due gravimetri sono stati tarati, essi si prestano - come si ̀̀ visto ottimamente a sostituire $i$ pendoli, sicché offrono un mezzo nuovo e veramente sicuro per $i$ collegamenti a largo raggio, ed in particolare per quelli fra stazioni nazionali di riferimento.

Si è presa perciò occasione dell'Assemblea Generale di Bruxelles 1951 dell'U.G.G.I., per collegare con la piì grande cura con Harzburg, e quindi indirettamente con Potsdam:

- anzitutto Uccle, dove già il valore della gravità nel sistema di Potsdam era noto con buona precisione $(\bar{j})$;

- per il tramite di Uccle: Parigi, Teddington, De Bilt e Kopenhagen (v. fig. 1);

- attraverso la catena delle stazioni pendolari di taratura in Germania: Trieste e Padova, stazione nazionale di riferimento italiana.

Il trasporto della gravità dalle stazioni tedesche ad Uccle ed a Padova è stato eseguito per via di terra, con lo stesso automezzo e con gli stessi criteri con cui sono state eseguite le operazioni di taratura in Germania; i collegamenti da Ulccle sono stati eseguiti per via aerea. A questo proposito, conviene osservare che con il tipo di gravimetri impiegati e per $\mathrm{i}$ collegamenti a grande distanza, l'aereo è il mezzo di trasporto ideale: gli strumenti non sono sottoposti a disagi, per cui la deriva si mantiene costante; ed i circuiti si possono chindere quasi sempre entro limiti di tempo molto brevi. Naturalmente, le limitazioni derivanti dagli orari delle linee aeree - e più ancora gli ostacoli spesso insormontabili frapposti dai doganieri! impongono molte volte di fare stazione nelle vicinanze degli aeroporti, o negli aereoporti stessi; ma questo non reca alcun pregiudizio ai collegamenti fondamentali, in quanto - come abbiamo già detto - il valore della gravità può essere trasportato facilmente, con l'approssimazione di qualche centesimo di mgal, dall'aereoporto ad un qualunque altro posto "vicino" (intendendo entro tali limiti differenze di gravità che non superino il campo della vite piccola, e distanze fino ad un paio d'ore di macchina; e intendendo sempre che siano rispettati i criteri di impiego sopra specificati al $\S 5, b$ ).

8. Risultati e confronti. - Riportiamo qui di seguito i risultati dei collegamenti sopra specificati, partendo dai valori corretti di $g$ nelle stazioni di taratura (v. tab. III). Per ogni stazione sono riportati 
anche $\mathrm{i}$ risultati dei collegamenti pendolari moderni, quali risultano dalle tre compensazioni (indipendenti):

- compensazione dello scrivente $\left({ }^{5}\right)$;

- compensazione di R. A. Hirvonen ( $\left.{ }^{6}\right)$;

- compensazione di S. Coron $\left({ }^{7}\right)$;

e dati successivi :

- collegamenti gravimetrici del Woollard $\left({ }^{8}\right)$;

- collegamenti gravimetrici del Martin $\left({ }^{9}\right)$, riferiti al valore (comune) di Uccle 981,132.0;

- collegamenti pendolari del Cook 1951 (inediti; da Harzburg).

$\dot{E}$ infine proposto un valore finale ragionato, con l'errore medio che, quando questo valore è basato essenzialmente sui collegamenti di cui qui si rende conto, è funzione anche dell'incertezza delle costanti di scala sopra indicate $(0,35 \% o)$.

I. - Uccle (Osservatorio Reale, pilastro della stanza gravimetrica).

\begin{tabular}{|c|c|c|c|c|c|}
\hline \multirow{2}{*}{ Stazione } & \multicolumn{4}{|c|}{ Differenze di gravità (mgal) } & \multirow{2}{*}{$\begin{array}{c}\mathrm{g}\left(\mathrm{cm} / \mathrm{sec}^{*}\right) \\
\text { (Potsdam= } \\
981,274.0)\end{array}$} \\
\hline & w. 50 & w. 52 & W. 52.W.50 & Media & \\
\hline $\begin{array}{l}\text { Euskirchen } \\
\text { Uccle }\end{array}$ & $+28.95 \div 0.12$ & $+28.79 \pm 0.11$ & -0.16 & $+28.87 \pm 0.12$ & $\begin{array}{r}981,103.15+021 \\
, 132.02+0.24\end{array}$ \\
\hline
\end{tabular}

Valori precedenti :

- compensazione Morelli: $981,131.5+0,8$;

- compensazione Hirvonen: 981,132.2 + 1,0;

- compensazione Coron: 981,131.2 0,4.

Come si vede, la concordanza è ottima; ciò consente di proporre con fiducia quale valore definitivo per Uccle (nel sistema di Potsdam):

$$
-U_{c c l e}=981132.0+0.2 \mathrm{mgal}
$$

Per le necessità dei collegamenti aerei, una stazione sussidiaria è stata istituita a Bruxelles all'aercoporto di Melsbroeck (cantina delle caldaie, di fronte alla caldaia centrale): 


\begin{tabular}{|l|l|r|r|r|r|}
\hline $\begin{array}{l}\text { Uccle } \\
\text { Melsbroeck }\end{array}$ & $+29.57 \pm 0.04$ & $+29.47 \pm 0.04$ & -0.10 & $+29.52 \pm 0.04$ & $981,132.02 \pm 0.24$ \\
$.161 .54 \pm 0.24$
\end{tabular}

Per lo stesso collegamento era stato trovato:

- da L. Jones, con gravimetro Norgaard (2 colleg.): $+29,44$ mgal;

- da J. Martin con gravimetro Western (2 colleg.): $+29,42$ mgal.

Prendiamo quindi come valore finale la media dei tre:

$$
\Delta \mathrm{g}=+29.46+0.03
$$

per cui il valore di $g$ all'aereoporto di Bruxelles sarà :

$$
\mathrm{g}_{\text {Melsbroeck }}=981,161.48 \div 0.24 \mathrm{mgal} \text {. }
$$

II a. - Teddington (Nat. Phys. Laboratory, Metrology Dept., pi-

\begin{tabular}{|c|c|c|c|c|c|}
\hline \multirow{2}{*}{ Stazione } & \multicolumn{4}{|c|}{ Differenze di gravità (mgal) } & \multirow{2}{*}{$\begin{array}{c}\text { g }\left(\mathrm{cm} \mid \mathrm{sec}^{\mathrm{2}}\right) \\
\text { Potsdam= } \\
981,274,0)\end{array}$} \\
\hline & W. 50 & w. 52 & W. 52.W.50 & Media & \\
\hline $\begin{array}{l}\text { Melsbroeck } \\
\text { Teddington }\end{array}$ & $+34.87 \pm 0.02$ & $+34.91 \div 0.03$ & +0.04 & $+34.89+0.04$ & $\begin{array}{r}981,161.48+0.24 \\
.196 .37 \pm 0.25\end{array}$ \\
\hline
\end{tabular}
lastro della misura assoluta nella stanza n. 11, al livello del pavimento).

Valori precedenti :

- compensazione Morelli : 981,196.1 +0,9;

- collegamento Martin: 981,196.2;

— collegamento Cook 1951: 981,196.1 $\div 0,5$.

Valore finale proposto:

$$
\mathrm{g}_{\text {Teddingtos }}=981,196.3+0.2 \mathrm{mgal}
$$

Misura assoluta $(*)$ :

- Clark 1937: 981,183.4 -- 0,000.6 (10).

Correzione al valore di Potsdam:

$$
\text { - 12,9 mgal. }
$$

(*) La misura assoluta essendo stata effeltuata a $75 \mathrm{~cm}$ di altezza dal pavimento, essa è stata qui ridotta alla quota di questo. 
II b. - Parigi (Osservatorio Astronomico, pilastro $P$ della nuova sala di gravità).

Nota: La descrizione delle varie stazioni di riferimento a Parigi, e nell'ambito dello stesso Osservatorio Astronomico, si trova nella pubblicazione di S. Coron $\left({ }^{7}\right)$. Le differenze fra i vari punti dell'Osservatorio sono state misurate con grande cura mediante gravimetri da J. Martin $\left({ }^{9}\right)$, sicché oggi è praticamente indifferente riferirsi ad uno qualunque di questi. I valori qui sotto riportati si riferiscono al pilastro $P$ sopra descritto.

\begin{tabular}{|c|c|c|c|c|c|}
\hline \multirow{2}{*}{ Stazione } & \multicolumn{4}{|c|}{ Differenze di gravità (mgal) } & \multirow{2}{*}{ 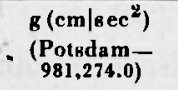 } \\
\hline & w. 50 & w. 52 & W. 52-W. 50 & Media & \\
\hline $\begin{array}{l}\text { Melsbroeck } \\
\text { Parigi (P) }\end{array}$ & $-217.63 \pm 0.01$ & $-217.71 \pm 0.03$ & -0.08 & $-217.67 \div 0.04$ & $\begin{array}{l}981,161.48+0.24 \\
980,943.81 \pm 0.25\end{array}$ \\
\hline
\end{tabular}

Valori precedenti :

- compensazione Morelli : 980,947.0 $+0,6$;

- compensazione Hirvonen: 980,944.4 $\div 0,6$;

- compensazione Coron: $980,943.53+0,31$;

— collegamento Woollard: 980,943.9;

- collegamento Martin (da Uccle): 980,943.9;

— collegamento Cook (attraverso Sèvres): 980,943.5 + 0.5.

Escluso il valore della compensazione Morelli (troppo alto perché basato anche sui collegamenti Parigi-Padova 1931 del cap. Reignier e Parigi-Madrid 1933 del prof. Sans Huelin ı, gli altri sono in ottimo accordo con quello sopra riportato. Sicché quale valore finale per Parigi sembra lecito proporre il seguente:

$$
\text { g }_{\text {Parigi } P}=980,943.8+0.3
$$

Esso coincide con la media dei precedenti, qualora si tenga conto della differenza di circa l'l\%o dei valori Martin (v. $\$ 9, b$ ), e corrisponde al valore $980,942.9$ per $i$ pilastri dell'antica sala gravimetrica.

La misura assoluta di Defforges 1883 , che aveva dato il risultato 980,943 , sarebbe quindi in perfetto accordo col valore attuale di Potsdam: ciò fa però dubitare della sua precisione (v. \$ 10 ).

Per le necessità più sopra indicate, una stazione sussidiaria è stata 
fissata in una delle stazioni del Martin $\left({ }^{9}\right)$ all'aereoporto Le Bourget, dogana lato arrivi (sotto la vetrata di separazione fra l'ufficio controllo passaporti e il hanco di controllo hagagli), dove è stato trovato il seguente valore:

$$
\text { o̊ I,o Bourgot }=980,949.81+0.25
$$

in perfetto accordo col valore trovato dal Martin.

Tenendo conto che la differenza fra Parigi $(P)$ e Sèvres (Bureau Internat. des Poids et Mesures, Pavillon du Breteuil, Punto $A$ ) è di + 3.23 + 0.01 mgal (Münck, 1951) il valore di $g$ a Sèvres diventa, nel sistema di Potsdam:

$$
g_{\text {Sovres }}=9 ; 0,440.6+0.3 \text {. }
$$

Misura assoluta $(h=65.93 \mathrm{~m})$ :

- Volet 1951: 980,916.

Correzione all'attuale valore di Potsdam:

$-24.6 \mathrm{mgal}$.

Il c. - Amsterdam (Aereoporto di Schiphol, a sinistra entrando

\begin{tabular}{|c|c|c|c|c|c|}
\hline \multirow{2}{*}{ Stazione } & \multicolumn{4}{|c|}{ Difforenze di gravita (mgal) } & \multirow{2}{*}{$\begin{array}{c}\left.\text { g(cm|sec }{ }^{2}\right) \\
(\text { Potadam= } \\
981,274.0)\end{array}$} \\
\hline & W. 50 & W. 52 & W. $52-W .50$ & Media & \\
\hline $\begin{array}{l}\text { Melsbroeck } \\
\text { Schiplıol }\end{array}$ & $+126.46 \pm 0.03$ & $+126.41+0.04$ & -0.05 & $+126.44+0.06$ & $\begin{array}{r}981.161 .48+0.24 \\
.287 .92 \div 0.25\end{array}$ \\
\hline
\end{tabular}
dalla pista, prima della dogana).

Altri collegamenti :

- Martin (da Uccle): 981,287.7.

La differenza Schiphol-De Bilt essendo stata determinata dal Martin in 19.0 mgal, ne risulta per il valore di g nel sistema di Potsdam:

$$
\mathrm{g}_{\mathrm{D} \text { B Bitt }}=981.268 .9+11.3 \text {. }
$$

Valori precedenti :

- compensazione Morelli: 981.268.0 $\dashv$ 0.7;

- compensazione Hirvonen: $981,267.9+0.6$;

- compensazione Coron: $981,267.9 \pm 0.4$;

- collegamento Woollard: 981,268.8;

- collegamento Martin: 981,268.7.

Valore finale proposto per De Bilt:

$$
\mathrm{E}_{\mathrm{Do} \mathrm{Bilt}}=181.268 .8 \pm 0.3
$$


II d. - Kopenhagen (Osservatorio dell'Istituto Geodetico, Buddinge, I'il. n. 1).

\begin{tabular}{|c|c|c|c|c|c|}
\hline \multirow{2}{*}{ Stazione } & \multicolumn{4}{|c|}{ Differonze di gravitù (mgal) } & \multirow{2}{*}{$\begin{array}{l}\mathrm{g}\left(\mathrm{cm} \mid \mathrm{sec}^{2}\right) \\
(\text { Potsdam }= \\
981,274.0)\end{array}$} \\
\hline & w. 50 & W. 52 & w. 52.W. 50 & Media & \\
\hline $\begin{array}{l}\text { Melsbroeck } \\
\text { Kopenhagen }\end{array}$ & $+396.30 \div 0.01$ & $+39586+0.03^{\circ}$ & -0.44 & $+396.08+0.22$ & $\begin{array}{r}981,161.48+0.24 \\
, 557.56 \pm 0.32\end{array}$ \\
\hline
\end{tabular}

Valori precedenti :

- compensazione Morelli : 981,558.4+ 0.7;

- compensazione Hirvonen: 981,557.5;

- compensazione Coron: $981,557.3+0.5$;

— collegamento Woollard: 981,556.9;

- collegamento Martin: 981,557.1.

- collegamento Trovaag - Jelstrup $\left({ }^{11}\right)$ : 981,558.9.

Valore finale proposto:

$$
g_{\text {Kopenhagen }}=981,557.5+0.4
$$

Esso coincide questa volta praticamente con la media di tutti i valori sopra riportati (che sono in buon accordo fra di loro), dato che per i gravimetri Worden n. 50 e n. 52 Kopenhagen è un po' al di fuori del campo di taratura (v. fig. 2) e dato che l'estremo superiore di questo è un po' meno preciso del resto $(v . . \$ 2 a)$.

III a. - Trieste (Stazione Sismica dell'Osservatorio Geofisico), Padova (Stazione nazionale italiana: sala gravimetrica al pianterreno della torre dell'Osservatorio Astronomico; $h=14,3 \mathrm{~m}$ ), Milano (Istituto di Geodesia del Politecnico, pilastro gravimetrico).

\begin{tabular}{|c|c|c|c|c|c|}
\hline \multirow{2}{*}{ Stazione } & \multicolumn{4}{|c|}{ Differenze di gravità (mgal) } & \multirow{2}{*}{$\begin{array}{l}\mathrm{g}\left(\mathrm{cm} / \mathrm{see}^{2}\right) \\
(\text { Potsdam- } \\
981,274.0)\end{array}$} \\
\hline & w. 52 & W. 52 & W. 52-W. 50 & Media & \\
\hline $\begin{array}{l}\text { Villaco } \\
\text { Trieste } \\
\text { Padova } \\
\text { Milano }\end{array}$ & $\begin{array}{l}+65.89+0.06 \\
-6.58 \pm 0.03 \\
-94.10 \div 0.02\end{array}$ & $\begin{array}{l}+65.84 \pm 0.09 \\
-6.55 \pm 0.03 \\
-94.08+0.03\end{array}$ & $\begin{array}{r}-005 \\
+0.03 \\
+0.02\end{array}$ & $\begin{array}{l}+65.88+0.14 \\
-6.57 \pm 0.04 \\
-94.09 \pm 0.03\end{array}$ & $\begin{array}{r}980,599.24+0.34 \\
.665 .12 \pm 0.36 \\
.658 .55+0.37 \\
.564 .46+0.38\end{array}$ \\
\hline
\end{tabular}


Valori precedenti :

\begin{tabular}{lcc} 
& Padova & \multicolumn{1}{c}{ Milano } \\
- compensazione Morelli : & $980,657.5+0.7$ & $980,566+3.0$ \\
- compensazione Hirvonen : & $980,657.0+0.7$ & - \\
- compensazione Coron: & - & $980.565 .94+0.57$ \\
- collegamento Martin (da Uccle): & & 980.565 .8
\end{tabular}

Valori finali proposti:

$$
\begin{aligned}
& \mathrm{g}_{\mathrm{Padova}}=980,658.6+0.4 \\
& \mathrm{~g}_{\text {Mileno }}=980,564.5+0.4
\end{aligned}
$$

Misura assoluta a Padova $(h=19,1 \mathrm{~m})$ :

- Lorenzoni (1885-86): 980,648.

Correzione che ne deriverebbe (tenendo conto che la differenza misurata fra le due stazioni a Padova è $0,90 \mathrm{mgal}$ ) all'attuale valore di Potsdam:

$$
-9.7 \mathrm{mgal} \text {. }
$$

III b. - Roma (Stazione pendolare nella cantina già dell'Istituto

\begin{tabular}{|c|c|c|c|c|c|}
\hline \multirow{2}{*}{ Stazione } & \multicolumn{4}{|c|}{ Differenza di gravita (mgal) } & \multirow{2}{*}{$\begin{array}{l}\text { (g cm!sec }) \text { ) } \\
\text { (Potsdam } \\
981,274.0)\end{array}$} \\
\hline & w. 50 & w. 52 & w. 52.w. 50 & Media & \\
\hline $\begin{array}{l}\text { Trieste } \\
\text { Roma }\end{array}$ & $-303.46 \div 0.05$ & $-303.68 \div 0.06$ & -0.22 & $-303.54 \pm^{0.12}$ & $\begin{array}{r}980,665.12 \pm 0.36 \\
, 361.58+0.38\end{array}$ \\
\hline $\begin{array}{l}\text { Padova } \\
\text { Roma }\end{array}$ & -297.10 & -296.82 & +0.28 & $-296.96 \pm 0.12$ & $\begin{array}{r}980,658.55 \pm 0.32 \\
, 361.59 \div 0.38\end{array}$ \\
\hline
\end{tabular}
di Topografia alla Facoltà di Ingegneria (ora dell'Istituto di Scienza delle Costruzioni), nell'angolo NNW dell'edificio $(h=49 \mathrm{~m})$.

Valori precedenti :

- compensazione Morelli: 980,366.3+0.8;

- compensazione Hirvonen: 980,366.3 + 1.1 ;

- collegamento Woollard (*): 980,364.1;

(*) Il valore pubblicato $\left({ }^{8}\right)$ dal Woollard $(980,363.1)$ è la media di 3 stazioni al piano terreno della Facoltà d'Ingegneria; la media delle differenze fra di esse e la stazione pendolare (misurate con il W. 50 ) ̀̀ $+1.02 \div 0.04$ mgal. 
- collegamento Cunietti (da Milano): 980,361.08;

- collegamento Boaga $\left({ }^{12}\right)$ : 980,360.4.

Valore finale proposto:

$$
\mathrm{g}_{\mathrm{Roma}}=980,361.6 \div-0.4
$$

Misura assoluta $(h=59 \mathrm{~m})$ :

- Pisati e Pucci (1882-87): 980,343.

Tenendo conto che la misura assoluta è stata eseguita nell'Istituto di Geodesia al $1^{\circ}$ piano della Facoltà l'Ingegneria, e che la differenza fra questo e la cantina gravimetrica (misurata col W. 50) è $+2.8 \mathrm{mgal}$, la correzione derivante all'attuale sistema di Potsdam è:

\section{- 15.8 mgal .}

9. Confronti con altre turature. - Collegamenti a grande distanza con gravimetri sono stati finora eseguiti dal Woollard e dal Martin.

a) Il Woollard, nel suo fondamentale lavoro $\left({ }^{8}\right)$ di collegamenti gravimetrici intorno al mondo, ha tarato il suo gravimetro su 19 fra le migrliori stazioni pendolari distribuite nell'emisfero settentrionale. La precisione della taratura è valutata nell' $1 \%$.

L'obiezione fondamentale al lavoro del Woollard riguarda la determinazione ed climinazione della deriva: egli infatti non chiudeva entro breve tempo i suoi circuiti, ma assumeva in generale quale deriva per un collegamento la media di quelle determinate ai due esterni di esso, all'inizio e alla fine delle misure. Però, come egli stesso ebbe a verificare e come abbiamo meglio mostrato successivamente $\left({ }^{1}\right)\left({ }^{2}\right)$, la deriva dei gravimetri Worden non è costante, per vari motivi non sempre precisabili (gradiente termico, stato di agitazione dello strumento, ecc.) e risente fortemente della variazione diurna della gravità, che quindi deve esser previamente calcolata ed eliminata. Perciò, per esempio, potremo facilmente avere al mattino, prima dell'inizio delle operazioni con il gravimetro, una deriva negativa, che potrà ritornare ad essere tale ( ${ }^{1}$ ) anche alla sera col ritorno del gravimetro in quiete; mentre per tutta la giornata di lavoro la deriva potrà anche essere fortemente positiva. $E$ evidente quindi che in questo caso la media dei due valori determinati sperimentalmente prima e dopo le operazioni di collegamento indurrà in errori molto forti (che potranno anche essere dell'ordine di $0,10 \mathrm{mgal} /$ ora). 
Se quindi il procedimento si estende a circuiti molto lunghi, come in qualche caso ha fatto il Woollard, non sono da escludere a priori errori anche dell'ordine del mgal.

In Europa però $i$ circuiti essendo stati chiusi in generale tutti entro breve tempo, ciò non si è verificato. Ed in quelle località in cui abbiamo rioccupato le stazioni del Woollard, l'accordo è sempre ottimo (entro $0,2 \mathrm{mgal}$ ). La differenza osservata fra gli estremi in comune è la seguente:

Kopenhagen-Roma : $\quad J \mathrm{~g}$ Woollard $=+1192.8 \mathrm{mgal}$

$$
\begin{aligned}
& \text { Morelli }=+1196.1 \mathrm{~s} \\
& \text { differenza }=-3.3 \mathrm{mgal}
\end{aligned}
$$

Come si vede, la differenza è molto forte. E però da tenere presente che il valore del Woollard a Roma è uno fra i meno sicuri fra tutti quelli dovuti a questo A., soprattutto per il fatto che non è stato rioccupato il posto esatto, e che il valore finale è la media di più stazioni non sicuramente rintracciabili. Un errore dell'ordine di 2 mgal non è perciò improbabile; e allora l'accordo fra le due differenze estreme rientra nell'ordine della precisione (sopra menzionata) connessa con le tarature dei differenti gravimetri impiegati.

b) Il Martin $\left({ }^{9}\right)$ ha effettuato la taratura dei suoi gravimetri (ben 8:3 Western e 5 Nortl-American) per confronto con tutte le differenze di gravità fra le stazioni pendolari che egli ha potuto rioccupare in Europa. Su un totale di oltre $10.000 \mathrm{mgal}$ complessivi per le differenze pendolari considerate, egli ha poi ricavato la costante di scala dei suoi gravimetri col metodo dei minimi quadrati.

Si vede però subito che, se le differenze pendolari considerate non sono solo quelle direttamente osservate e se in tale calcolo intervengono molte differenze di gravità fra stazioni pendolari di differenti Paesi, per ogni coppia di esse che si trovi in queste condizioni l'errore connesso con i valori delle stazioni di riferimento è di natura sistematica. Ciò può quindi influire sul risultato della taratura.

Riportiamo ora assieme $i$ valori ottenuti dal Martin e dallo scrivente nelle stazioni di riferimenti in comune:

\begin{tabular}{|l|r|r|r|}
\hline \multicolumn{1}{|c|}{ Stazione } & Martin & Morelli & \multicolumn{1}{c|}{$\Delta$} \\
\hline & & & \\
\hline Kopenhagen & $981,557.1$ & $981,557.6$ & -0.5 \\
De Bilt &, 268.7 &, 268.9 & -0.2 \\
Uccle &, 132.0 &, 132.0 & 0.0 \\
Parigi $(P)$ & $980,943.9$ & $980,943.8$ & +0.1 \\
Milano &, 565.8 &, 564.5 & +1.3
\end{tabular}


Tali differenze sono riportate graficamente nella fig. 4.

Anche considerando un'imprecisione maggiore nelle stazioni dello scrivente a Kopenhagen (perché fuori del campo di taratura; v. fig. 2) ed a Milano (perché ottenuto per numerosi trasporti successivi; v. § 8, IIl a), l'errore sistematico derivante dagli errori nelle costanti di scala delle due tarature è molto probabilmente dell'ordine di $1 \%$. Ricordando che la precisione della taratura dello scrivente è del $0,35 \%$ ( $\$ 4$ ) e che l'imprecisione delle differenze fra gli estremi è del $0,5 \%$, ne ri-

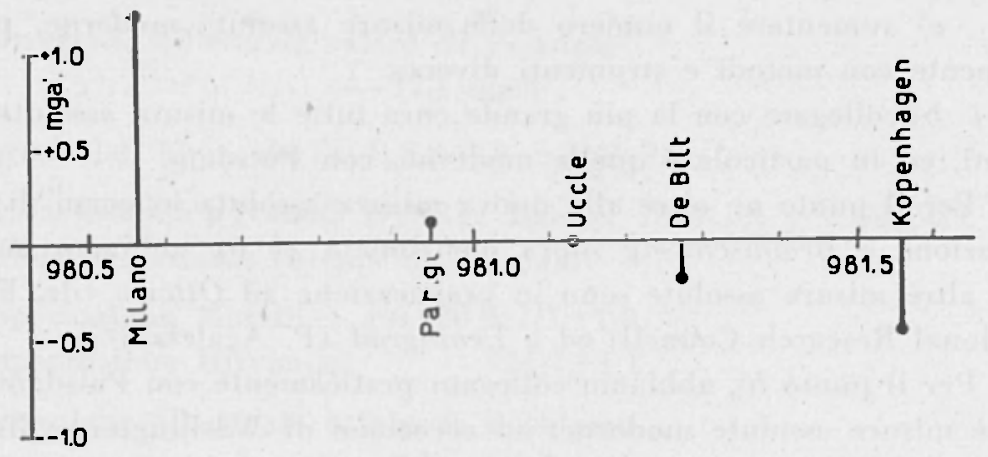

Fig. 4 - Differenze Martin-Morelli.

sulterebbe che la precisione della taratura del Martin è pure molto elevata, anche se presenta una piccola differenza sistematica con quella dello scrivente. Solo un confronto diretto su basi in comune più sicure potrà però consentire di precisare questa differenza.

10. Correzione al valore di Potsdam. - Tutti i valori di gravità sono oggi basati, per una decisione dell'Associazione Intern. di Geodesia presa nell'Assemblea Generale di Londra 1912, sulla misura assoluta di Potsdam $(981,274+0,003)$.

Le misure assolute successive (Washington e Teddington), collegate con Potsdam, hanno indicato però che il valore di Potsdam è molto probabilmente affetto da un notevole errore. Già nel 1946 lo scrivente aveva perciò proposto $\left({ }^{13}\right)$ di sostituire al " sistema di Potsdam " un " sistema internazionale ", basato ciò̀ sui risultati non di una sola misura assoluta, ma di tutte le misure assolute finora effettuate, o meglio di quelle moderne più sicure.

Successivamente il Berroth (1948), rielaborando i calcoli della misura assoluta di Potsdam, trovò $\left({ }^{1 *}\right)$ che le correzioni apportate da Kühnen e Furtwangler per tener conto della forma dei coltelli e delle 
loro deformazioni elastiche, non erano complete, e che il risultato della misura assoluta andava così modificato:

$$
g_{\text {Potsdain }}=981,261+0.001 \text {. }
$$

Il valore di Potsdam è dunque certamente shagliato, e l'errore non è trascurabile. L'adozione del "sistema internazionale " risulta quindi giustificata.

Per la determinazione sicura e definitiva del termine correttivo, occorre:

a) aumentare il numero delle misure assolute moderne, possibilmente con metodi e strumenti diversi;

b) collegare con la più grande cura tutte le misure assolute esistenti, ed in particolare quelle moderne, con Potsdam.

Per il punto $a$ ), oltre alla nuova misura assoluta in corso di preparazione a Braunschweig sopra menzionata $(\$ 6)$, abbiamo notizia che altre misure assolute sono in preparazione ad Ottaua (dr. Field, National Research Council) ed a Leningrad (P. Agaletzki).

Per il punto $b$ ), abbiamo collegato praticamente con Potsdam tutte le misure assolute moderne, ad eccezione $\mathrm{di}$.Washington e di $\mathrm{Ot}$ tawa (che però rientrano nel campo di taratura dei nostri gravimetri, v. fig. 2; e che quindi potrebbero essere pure incluse in questi collegamenti) e di Leningrad (che col valore 981,929 non rientra nel campo di taratura).

Riassumiamo qui di seguito le correzioni al valore attuale di riferimento per il sistema di Potsdam che derivano dalle misure assolute moderne:

\begin{tabular}{|c|c|}
\hline Misure assolute & Correzione \\
\hline Potsdam (Berroth) . . & $-13.0 \mathrm{mgal}$ \\
\hline Washington ... & -17.1 \\
\hline Leningrad & $-2.4(?) "$ \\
\hline Teddington & -12.9 \\
\hline Parigi (Volet) & -24.6 \\
\hline
\end{tabular}

Le correzioni da Teddington e Parigi risultano dal $\$ 8$; quelle da Washington e Leningrad sono state ricavate come segue:

Washington (Nat. Bur. of Standards, $h=95 \mathrm{~m}$ ):

- Misura assoluta (Heyl e Cook, 1928-1936): $g=980,0816-0,0012\left({ }^{10}\right)$. 
Collegamenti pendolari:

1933 Brown, con Potsdam

1939 Brownc e Bullard, da 'T'eddington

Collegamenti con gravimetro:
980,0998

980,0978

980,0985

980,0987

980,0985

Valore nell'attuale sistema di Potsdam $=\overline{980,0987 \div 0.3}$.

Correzione all'attuale valore di Potsdam:

- $17.1 \mathrm{mgal}$.

Leningrad (Ist. Metr. de I'U.R.S.S.: $h=4 \mathrm{~m}$ ?).

- Misura assoluta (Ivanoff 1906-11; Baskov 1933-...): $g=981,929$.

Valori nell'attuale sistema di Potsdam:

— compensazione Morelli : $g=980,931.1+1.0$

- compensazione Hirvonen: $\quad, 931.7+1.1$

Correzione all'attuale valore di Potsdam: -2.4 mgal ?

Per le misure assolute antiche, la correzione è riassunta nel prospetto che segue:

\begin{tabular}{|c|c|}
\hline Misura assoluta & Correzione \\
\hline Roma & $-15.8 \mathrm{mgal}$ \\
\hline Madrid & $-0.4 D$ \\
\hline Parigi (Defforges) . . . & $+0.1 D$ \\
\hline Vienna & $+6.1 \mathrm{D}$ \\
\hline ..... & $-9.7 \nu$ \\
\hline Monaco & +3.0 D \\
\hline
\end{tabular}

La correzione per Monaco risulta dal $\S 6$; quelle per Roma, Parigi e Padova dal $\$ 8$. Le correzioni per Madrid e Vienna sono state ricavate come segue:

Madrid (Osservatorio Astronomico, pilastro della Biblioteca; $h=$ $=656 \mathrm{~m}$ ).

— Misura assoluta (Barraquer, 1882): $g=979,981$. 
Valori nell'attuale sistema di Potsdam:

- compensazione Morelli:

- compensazione Hirvonen:

$g=979,983.0$

- compensazione Coron:

- collegamento Martin:

Valore finale proposto:

$$
g=979,981 \cdot 4 \pm 0,4
$$

Correzione all'attuale valore di Potsdam:

$$
\text { - 0.4 mgal. }
$$

Vienna (Osservatorio Astronomico, Wahring, Türkenschanze, sotterraneo Nord; $h=236 \mathrm{~m}$ ).

- Misura assoluta (von Oppolzer, 1884): $g=980,859$.

Valori nell'attuale sistema di Potsdam:

- compensazione Morelli : $\quad g=980,8529 \div 0.8$

- compensazione Hirvonen: $\quad, 8528-0.8$

Correzione all'attuale valore di Potsdam:

$$
+6.1 \text { mgal. }
$$

Le misure assolute antiche sono indubbiamente meno precise di quelle moderne (soprattutto per le imperfette determinazioni degli effetti dei coltelli, della oscillazione del supporto e della flessione dei pendoli, per le più imprecise determinazioni di tempo, ecc.). Però anche dalla tabellina delle misure moderne si vede che la precisione conseguibile non è stata finora superiore a qualche mgal.

Ciò dimostra ancora una volta l'opportunità di basare il nuovo sistema di riferimento per le misure di gravità non su una sola, ma sul maggior numero possibile di misure assolute (moderne); e comprova così la necessità del " sistema di riferimento internazionale " proposto dallo scrivente.

La sua adozione definitiva è subordinata, conformemente alla risoluzione della IX Assemblea Generale dell'Uinione Geodetica e Geofisica Internazionale, all'ultimazione della nuova misura assoluta in corso di esecuzione a Braunschweig, ed eventualmente di quelle a Ottawa e Leningrad; nonché al completamento delle esperienze della nuova misura assoluta iniziata dal Volet a Parigi.

Intanto si può assumere come valore provvisorio quello che risulta dalla media delle misure assolute moderne:

$$
-14.0+36 \text { ingal }
$$


L'entità dell'errore medio dimostra ancora una volta la necessità di misure assolute moderne veramente precise.

Provvisoriamente si avrà quindi:

Sistema internazionale $=$ Sistema di Potsdam $-0,014 \mathrm{~cm}$. $\mathrm{sec}^{-2}$.

Ci sia lecito qui far notare che delle misure assolute antiche, quelle che meglio si accordano con le moderne sono proprio quelle italiane; il valore medio della correzione derivante all'attuale sistema di Potsdam dalle misure assolute di Roma e Padova è infatti - $13 \mathrm{mgal}$.

11. Conclusioni. - I collegamenti di cui qui si è reso conto non pretendono naturalmente di essere definitivi: il loro scopo era solo quello di portare un contributo alla istituzione di una rete gravimetrica europea, e di mostrare in quali condizioni e con quali criteri $i$ gravimetri possano effettivamente sostituire, con grandi vantaggi, $i$ pendoli.

Il " mgal europeo" che è risultato dalle operazioni di taratura di cui qui si è reso conto ha indubbiamente una precisione molto elevata, quale oggi non era ancora dato di poter disporre. Essa potrà anche essere naturalmente - e sperabilmente - superata nel futuro, e soprattutto il campo di taratura potrà essere esteso, quando si rendano disponibili nuove stazioni pendolari di alta precisione opportunamente distribuite in latitudine (o altitudine): a noi interessava qui, fra l'altro, indicare la via, e indicare con quali criteri i pendoli possono ancora essere preziosi ed indispensabili per i gravimetri; e come questi poi li superino nella pratica applicazione $e$ in particolare servano addirittura indirettamente a controllare la precisione di quelli.

Interessante sareble ora poter utilizzare i gravimetri così tarati per più numerosi collegamenti a largo raggio, ed in special modo effettuare un confronto fra il " mgal europeo ", qui ricavato, col cosiddetto " mgal americano ", in corso di istituzione ad opera dell'U.S. Coast and Geodetic Survey.

Trieste - Osservatorio Geofisico dell'Istituto Nazionale di Geofisici - novembre 1951.

\section{RIASSUNTO}

Per $i$ due gravimetri Worden n. 50 e n. 52 la determinazione della costante di scala è stata effettuata per confronto con 18 stazioni pendolari tedesche, entro un campo di 1500 mgal (da 980,00 a 981,50: praticamente corrispondente all'Europa centro-meridionale). Ė risulta- 
to che le costanti fornite dalla Casa costruttrice avevano un errore di circa il $2 \%$. Le nuove costanti risultano lineari, nel campo considerato, con un errore del $0,35 \%$. Ciò consente di utilizzare con vantaggio $i$ gravimetri in questione, sempre nel campo di taratura e con opportuni criteri d'impiego, in sostituzione dei pendoli, con un errore medio "reale" inferiore al $0,5 \%$. Se ne è fatto impiego per collegare, indirettamente, con Potsdam le stazioni nazionali di riferimento di Uccle (981,132.0 \pm 0.2), Parigi (980,943.8 0.3), De Bilt (980,268.8 0,3), Kopenhagen $(981,557.5 \pm 0.4)$, e Padova $(980,658.6 \pm 0.4)$, nonché le misure assolute di Teddington (981,196.3 \pm 0.2$)$, Monaco $(980,733.0 \pm$ + 0.4), Braunschweig $(981,265.5$ i- 0.1$)$ e Roma $(980,361.6$ i- 0.4). La correzione provvisoria all'attuale (sistema di Potsdam " viene così confermata in $-13.9 \pm 3.5 \mathrm{mgal}$.

\section{BIBLIOGRAFIA}

(1) MoRelli C.: Rilievo gravimctrico e riduzione isostatica nell'talia nordorientale. Tecnica Italiana, Nuova Serie, VI, 3 e 4, Trieste 1951.

(2) Morflur C.: Studio del gravimetro Worden n. 50 e sua applicazione per un rilievo geofisico di dettaglio alle foci del Timavo. Ann. di Geof., IV, 2, Roma 1951.

(3) WeIKf.N K.: Ergebnisse der Pendelmessungen der Jahre 1934 bis 1943. Veroff. d. Geod. Inst. Potsdam, n. 3, 1950.

(4) Morelli C.: Collegamento gravimetrico Padova-Trieste e rilievo gravimetrico regionale del Veneto centro-orientale. Riv. di Genfis. appl., XI, 2, Milano, 1950.

(5) Morelli C.: Compensazione della rete internazionale delle stazioni di riferimento per le misure di gravità relativa. Boll. Soc. Adriatica Scienze Nat., vol. XLI, Trieste 1946.

(6) HiRvonen R. A.: On the establishment of the values of gravity for the national reference stations. Publ. Isostatic Institute n. 19, Helsinki 1948.

(7) Coron S.: Valeur de la pesanteur à Paris déterminée a l'aide des liaisons internationales européennes. Bull. Géod., n. 16, 118-139, juin. 1950.

(8) Woollard G.: The gravity meter as a geodetic instrument. Geophysics, $15,1-29,1950$.

(9) Martix J.: Liaisons gravimetriques internationales 1947-1951 (in corso di pubblic.).

(10) JefFreYs H.: On the absolute measurement of gravity. M.N.R.A.S., Geoph. Suppl., 5, n. 9, 398, 1949.

(11) Trovade e Jelstrup: Gravity comparisons Oslo-Teddington-Stockholm Kopenhagen. Den norske Gradmalingskom. on Norges geograf. Opmaling, Oslo 1950.

(12) Boaga G, Tribalto G. e Zaccara G.: Campagna geo-gravimetrica effettuata nel giugno 1950. Quaderno n. 2, Sezione Geofisica dell'Ufficio Geologico, Roma 1950.

(13) Morelli C.: Per un sistema di riferimento internazionale delle misure di gravità. Nota preliminare. Geofisica pura ed appl. v. VIll, f. 3-4, Milano 1946.

(14) Ветroth A.: Das Fundamentalsystem der Schwere im Lichte neuer Reversionspendelmessungen. Bull. Gèod., n. 12, Paris 1949. 